\title{
Analysis on the Psychological Health Education from the Perspective of Narrative Therapy
}

\author{
Li Jing \\ Yunnan University of Business Management, Kunming, Yunnan, 650106
}

Keywords: Psychological health education; narrative therapy; social cognition; analysis

\begin{abstract}
Psychological health education has always been important in the social development, and more new methods have been adapted to practical psychological health education in which they have positive effects with the constant development of modern society. Narrative therapy, as one post-modern psychotherapy method, is combined with psychological health education, greatly promoting the entire level of modern mental health education. However, due to the lack of specific practical experience, it hasn't been widely promoted and applied in its practical application. This paper analyzes the current situation of narrative therapy and its application mode in mental health education, and education workers should make innovations while deepening the research on narrative therapy.
\end{abstract}

\section{Introduction}

The so-called narrative therapy means a new psychological therapy based on modern psychotherapy, which plays a very positive role in the promotion of modern psychotherapy. And psychological health education from the perspective of narrative therapy mainly means respecting local culture, paying attention to people's cognition with society and emphasizing the analysis of mental problems from the social culture. It is a new psychological health teaching method that separates people from the problem by means of externalized worldview. Only by adopting corresponding intervention methods on the basis of keeping narrative worldview in the process can the psychological health education level from the perspective of narrative therapy be truly and comprehensively promoted.

\section{Overview of Narrative Therapy}

\subsection{Definition of narrative therapy}

Different from traditional psychotherapy, narrative therapy adopts the method of "storytelling", "problem externalization" and "from thick to thin", which not only breaks the traditional concept of regarding people as the problem, but has higher promotion value in the operability and effect.

This concept was originated from the 1960s and 1970s and affected by the contemporary thought and social activities, mostly influenced by the philosophical thinking of Foucault and Derrida. Family therapy was formally founded in the 1980s, whose founder is Michael White and David Epston, two experts at family therapy. The theory source of the method is mainly the post-modern philosophical thinking, and its main therapy concept is equal, open and diversified ${ }^{[1]}$. Once put forward, it has played a significant role in family therapy.

\subsection{Main application range of narrative therapy}

Narrative therapy was mainly applied in the family therapy at first. But after years of development and the social work background of originators, it was applied to other fields. White applied narrative therapy to the community work and started corresponding practice in the 1990s. In the actual practice, the theory and technique of narrative therapy are combined with specific characteristics of community work. Community people are guided to conclude the methods and skills for specific problems to realize the thinking with value outlook and dream. Finally, it will promote the connection between people and people, community and community.

\subsection{Narrative therapy and the analysis of psychological health education}

Mental health education is always helping individuals, which is not to make individual cognize and learn the "objective and scientific" standards of mental health, but to help them master the experiment 
and skills for various mental problems, as well as form independent personal quality and specific value outlook by means of the corresponding education. It has the same basic goal as narrative therapy, namely, the application of narrative therapy to the mental health education is of great positive significance to promoting the entire level of mental health education ${ }^{[3]}$.

\section{Overview of Narrative Psychological Health Education}

\subsection{Psychological health workers' role orientation from the perspective of narrative therapy}

Due to its basis on the post-modern philosophy, everyone is regarded as the expert of his own life in the practical therapy application, and all of them have life knowledge and survival skills that are acquired or concluded from their life experience. Therefore, as for the mental health education workers from the perspective of narrative therapy, their roles have gradually transformed from original knowledge provider to the cooperator and recorder of education ${ }^{[4]}$. Therefore, the related education content, model and methods have changed a lot due to the change of these workers' roles.

\subsection{Specific content of psychological health education from the perspective of narrative therapy}

Narrative therapy is different from traditional one, so the mental health education content from the perspective of narrative therapy has changed a lot. Specifically, it has become the interpretation about mental health problem of the group or individual for themselves, not the original interpretation made by the external experts. It is the change of role orientation that the related content is not so-called "expert knowledge" any longer, but the local knowledge based on specific social culture and community content. In other words, mental health education workers adopt the method of questioning, interview or structured activity to help individuals form the ability to develop and conclude indigenous knowledge in specific experience $^{[5]}$.

\subsection{Psychological health education methods from the perspective of narrative therapy}

Now that the role orientation and specific content of mental health education from the perspective of narrative therapy have changed a lot, the working methods also have changed in the practical work. Currently, the most common method in the practice is to use therapy documents. The detailed form is that the attending doctors document the indigenous knowledge developed in the interview with patients and then help people form their distinct lives more positively and actively ${ }^{[6]}$. And as for the mental health education from the perspective of narrative therapy, the specific method is basically similar with that of narrative therapy, mainly through documentation. In addition, related workers should combine with people's specific experience and personal habits in the actual application, conclude relevant mental health knowledge and experience by means of letters, response manual, audio and video, and help other people with similar problems on this basis to achieve corresponding mental health education.

\subsection{Cognition with psychological problems from the perspective of narrative therapy}

The cognition with mental problems from the perspective of narrative therapy mainly consists of the following aspects.

The cognition from the narrative therapy varies from the traditional one, tending to thinking the mental problem based on social culture. Generally, the mental health education from the perspective of narrative therapy assumes that there are mainly two conditions of mental problems: the first one is that individual's mental problem because he cannot satisfy some standards of social culture; and the other one is the mental problems appeared in the process of meeting some social culture standards.

The externalization of concepts. It means that people and problem should be separated from each other although they have some relationships ${ }^{[7]}$. Such concept not only makes the objectification of mental problems but makes its combating practice with the objectification of people, which is helpful to better find and solve problems. And for the mental health education from the perspective of narrative therapy, the externalization of concepts can separate people from the problems, making individuals have energy and spirit to face their own mental problems. 


\section{Practice Model Analysis of Mental Health Education from the Perspective of Narrative Therapy}

The real practice of mental health education mainly includes two stages. The first one is preparation where the related staff should establish good cooperation with objects of psychological education. And the second one is practice which means that the staff should carry out specific activities according to the habits of its specific objects, which is also the most important stage in the whole process. And the model of current practice mainly consists of the following types.

\subsection{The questioning pattern}

The questioning pattern is the most representative one in the mental health education from the perspective of narrative therapy, originated from the United States, and its main model in the practical application is to help people acquire the knowledge and experience to solve problems though the combination of big questions and field interview. In general, the time of questioning is about 1.5 to 2 hours, and big questions are about 1 to $5^{[8]}$. It should be noticed that problems should cover various ranges to guarantee all the individuals in the field have the ability to respond. As far as the current psychological health education, the questioning types can be divided into two kinds. The first one is questioning with the function of influence, such as the externalized questioning, developing preferred stories, and concluding knowledge and skills. The other one is that with the function of reflection, which mainly happens after the first one, helping the individual or community conclude the experience to finally realize the orderly accomplishment of psychological health education. For example, in the process of psychological health education, related staff can randomly select one person to ask some questions after finishing the first part, for instance, "which story or viewpoint has given you a deep impression in the sharing process?”. Then the worker can have a simple interview with the answer and make corresponding recording. At the end, they can arrange and summarize the knowledge and experience based on the specific recording content, and then make the corresponding documents for better communication.

\subsection{The pattern of structured group activities}

The pattern of structured group activities, common in the related practice, is based on the collective narrative practice method, which integrates people, nature, sports, history with metaphors and customs about culture, and establishes a structured group activity. Lots of metaphors are usually be applied to the practice. Mental health education from the perspective of narrative therapy will be accomplished by likening different images in the structure to diversified mental states. In general, there are many structured constituted factors, such as time structure, space structure and character structure. Corresponding model should be selected based on people's specific conditions and actual demands in the practical application ${ }^{[9]}$. Only by this way can its positive role in the practice of psychological health education be manifested.

\subsection{The pattern of health education}

Health education model, based on the narrative therapy, has the similar content with mental health education, but has its own development model. Psychological health workers usually need to record and arrange the stories; knowledge and skills shared or described by people in the question, and then make them into corresponding psychological health education files after the practice. Files can have various forms, such as the audio, video and word. Select the most appropriate document based on the individual's practical condition and develop specific mental health education ${ }^{[10]}$. It can not only effectively ensure the smooth development of mental health education but also promote the specific effects, having a very positive influence on solving the mental problems of individuals or groups.

\section{Conclusion}

In summary, narrative therapy, as the most representative post-modern psychotherapy, has a very positive role in the innovation of psychotherapy method and promoting the therapy level for patients with mental problems. Even though the current related work has made a great progress, it still needs the further 
research and innovation about intervention methods along with the social development, to truly realize the entire improvement of modern psychological education.

\section{References}

[1] Liu Shumin, Du Jian, Wang Hao and Yang Qiuli. Prevention and treatment of psychosomatic diseases from the perspective of narrative medicine[J]. Modern TCM Clinic, 2018, 25(01):19-22.

[2] Zhu Hui. The application of narrative therapy in the grief counselling of bereaved patients after the earthquake[J]. China Social Work, 2017(30):28-29.

[3] He Fei. The application of narrative therapy in the case of depression[J]. Journal of ChongQing City Management College, 2017, 17(03):1-3+17.

[4] Wen Wenxiang. An attempt of narrative psychotherapy[J]. Mental Health Education in Primary and Middle School, 2017(21):52-54.

[5] Fei Junfeng and Zhao Zhao. Mental health education from the perspective of narrative therapy[J]. Medicine and Philosophy(A), 2017, 38(03):52-55.

[6] Wu Limin and Ma Yanjie. The application of narrative therapy in college mental health education[J]. Campus Life \& Mental Health, 2016, 14(03):204-206.

[7] Huang Xinxin. Analysis of the therapeutic mechanism of narrative psychotherapy[J]. Journal of Hubei Correspondence University, 2016, 29(07):95-96.

[8] Zhao Kuaile, Li Xiangyan, Jiang Li and Zhao Tiyu. Overview of narrative therapy and its application[J]. Journal of Nursing, 2016, 23(04):34-36.

[9] Zhao Zhao. Narrative therapy documents and its indigenous application[J]. Medicine and Philosophy(B), 2015, 36(11):72-75.

[10] Gao Jingjing. The enlightenment of narrative psychotherapy on adolescent mental health education[J]. Folk Psychology, 2015(08):45-46. 